\title{
Efectos del vendaje Low-Dye sobre el pie. Revisión narrativa
}

\author{
Effects of the Low-Dye tape on the foot. A narrative review \\ Óscar Madruga Armada, Raúl Juan Molines Barroso y Ángel Manuel Orejana García \\ Clínica Universitaria de Podología. Facultad de Enfermería, Fisioterapia y Podología. Universidad Complutense de Madrid. Madrid. España
}

\begin{abstract}
Palabras clave:
Vendaje atlético, vendaje ortésico, pronación, aparatos ortopédicos, marcha, cinemática, cinética, tape, Low Dye Tape.
\end{abstract}

Keywords:

Athletic tape, orthotic bandage, pronation, orthotic devices, gait, kinematics, kinetics, taping, Low Dye Tape.

\section{Resumen}

El vendaje Low-Dye se considera el gold estándar entre las técnicas de vendaje para el control de la caída del arco longitudinal del pie, según la literatura. Dicha técnica de vendaje ha recogido la atención de numerosos estudios en los últimos años, en los cuales se ha evaluado sus efectos mecánicos y clínicos.

Cinéticamente, los ensayos clínicos recogidos coinciden en que genera una disminución de las presiones plantares en la región medial del antepié y su aumento en la región lateral del mediopié. Cinemáticamente, el estudio del arco longitudinal interno ha centrado la atención de un mayor número de autores, debido a la localización anatómica del vendaje Low-Dye. El principal efecto cinemático producto de la colocación del vendaje Low-Dye consiste en una disminución en la caída del arco longitudinal interno. Finalmente, a nivel clínico destaca la mejoría del dolor en pacientes con fascitis plantar mediante el uso del vendaje Low-Dye.

En conclusión, los efectos cinéticos y cinemáticos observados guardarían relación con una disminución del momento de fuerza pronador neto que soporta el pie, así como el momento de flexión dorsal neto que soportan las articulaciones de la columna interna y podría favorecer la instauración del mecanismo de Windlass. Al favorecerse el mecanismo de Windlass, el estrés tensil que soportan diversas estructuras, como la fascia plantar, disminuye, mejorando así el dolor relacionado con esta patología según la bibliografía. Pese a ello es necesario la realización de más ensayos que evalúen los efectos de este vendaje.

\begin{abstract}
The Low-Dye tape is considered the gold standard among the tape techniques for the control of the fall of the longitudinal arch of the foot according to the literature. This tape technique has attracted the attention of numerous studies in recent years, in which its mechanical and clinical effects have been evaluated.

Kinetically, the collected clinical trials coincide in the generation of a decrease in plantar pressures in the medial region of the forefoot and its aument in the lateral midfoot region. Kinematically, the study of the internal longitudinal arch has focused the attention of a greater number of authors, due to the anatomical location of the Low-Dye tape. The main kinematic effect produced by the placement of the Low-Dye tape consists in a decrease in the fall of the internal longitudinal arch. Finally, at a clinical level, the improvement of pain in patients with plantar fasciitis is highlighted by the use of the Low-Dye tape.

In conclusion, the kinetic and kinematic effects observed would be related to a decrease in the net moment of pronation force that supports the foot as well as the net moment of dorsal flexion that support the joints of the internal column and could favor the establishment of the Windlass mechanism. By favoring the mechanism of Windlass, tensile stress that support various structures, such as the plantar fascia, decreases, improving pain related to this pathology according to the literature. Despite this, it is necessary to carry out more trials to evaluate the effects of this bandage.
\end{abstract}




\section{INTRODUCCIÓN}

El vendaje terapéutico es una intervención utilizada comúnmente para reducir el dolor, controlar el movimiento de las articulaciones, mejorar la propiocepción y normalizar el tono muscular. La rigidez del vendaje permite el cambio mecánico en la posición de la articulación 1 .

Una de las condiciones biomecánicas más comunes que pueden requerir abordaje mediante un vendaje funcional va a ser el exceso de pronación en el pie. La pronación del retropié es un movimiento complejo, fisiológico, que ocurre durante la fase de apoyo medio de la marcha. En esta fase del ciclo de la marcha tienen lugar diversos movimientos de estructuras óseas y articulares que conducen a la pronación del pie y a una disminución del arco medial. Así, tiene lugar una eversión del calcáneo, una aducción y flexión plantar del astrágalo, una pronación del escafoides y una rotación interna de la tibia².

La técnica de vendaje Low-Dye ha sido un foco importante de investigaciones relacionadas con el arco longitudinal del pie y se considera el gold estándar entre las técnicas

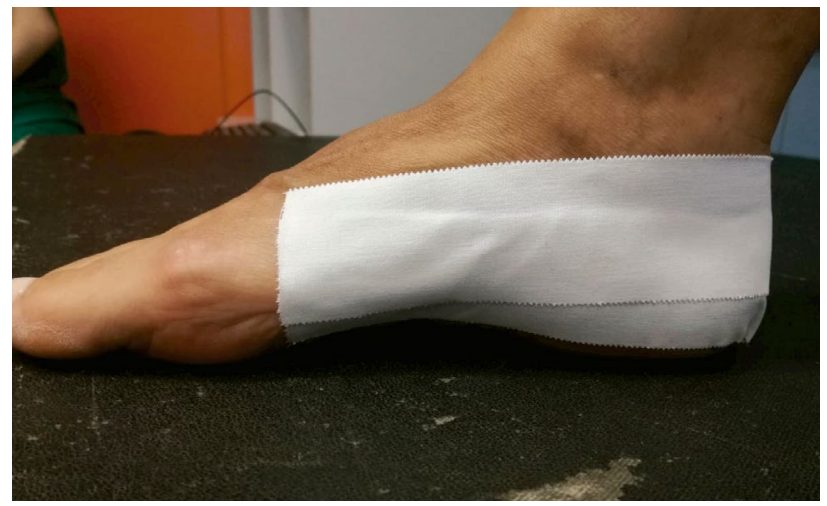

Figura 1. Vendaje Low-Dye. Posicionamiento de las tiras de anclaje desde una visión sagital.

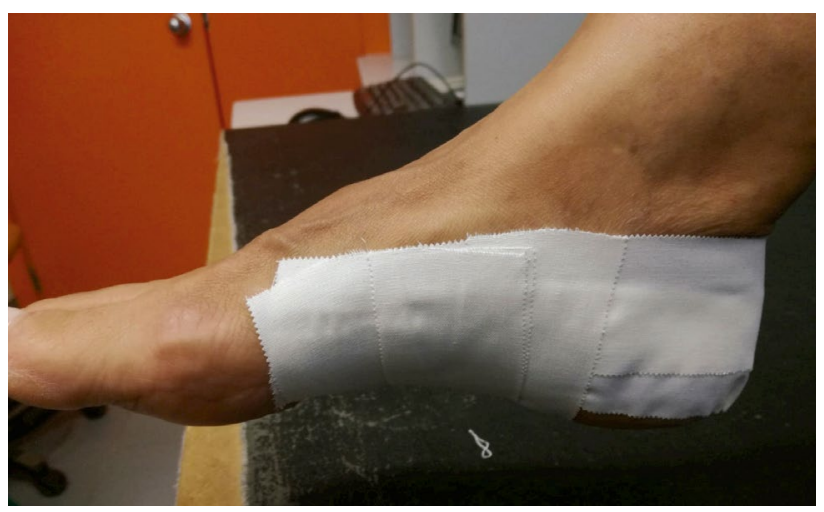

Figura 2. Vendaje Low-Dye. Tiras activas desde una visión sagital. de vendaje para el control de la caída del arco longitudinal del pie ${ }^{3}$.

La técnica de vendaje Low-Dye fue descrita originalmente por el doctor Ralph Dye en 1939 (Figuras 1-5) 4 . El principio de este vendaje consiste en fijar el eje de la articulación subastragalina, y fue realizado con el objetivo de reducir el exceso de pronación y aliviar los trastornos derivados de este movimiento, como el dolor o patologías como la fascitis plantar, tendinopatías del tendón tibial posterior, tendinopatías del tendón de Aquiles o el síndrome de estrés tibial medial 2,3,5.

Existen diversos grados de rigidez en las vendas a la hora de realizar un vendaje. Así, contamos con diversas técnicas de vendaje. La técnica rígida se basa en el uso de un material rígido a fin de inhibir un movimiento doloroso, siendo una técnica preventiva. La técnica blanda o suave también busca limitar el movimiento lesivo, aunque para su realización se usan vendas elásticas, obteniendo así una limitación menor del movimiento. Consistiría en una técnica terapéutica. Por otro lado, contamos con la técnica semirrígida, la cual se basaría en la utilización de ambos tipos de vendas a fin de limitar la función (en mayor escala que con la técnica blanda)

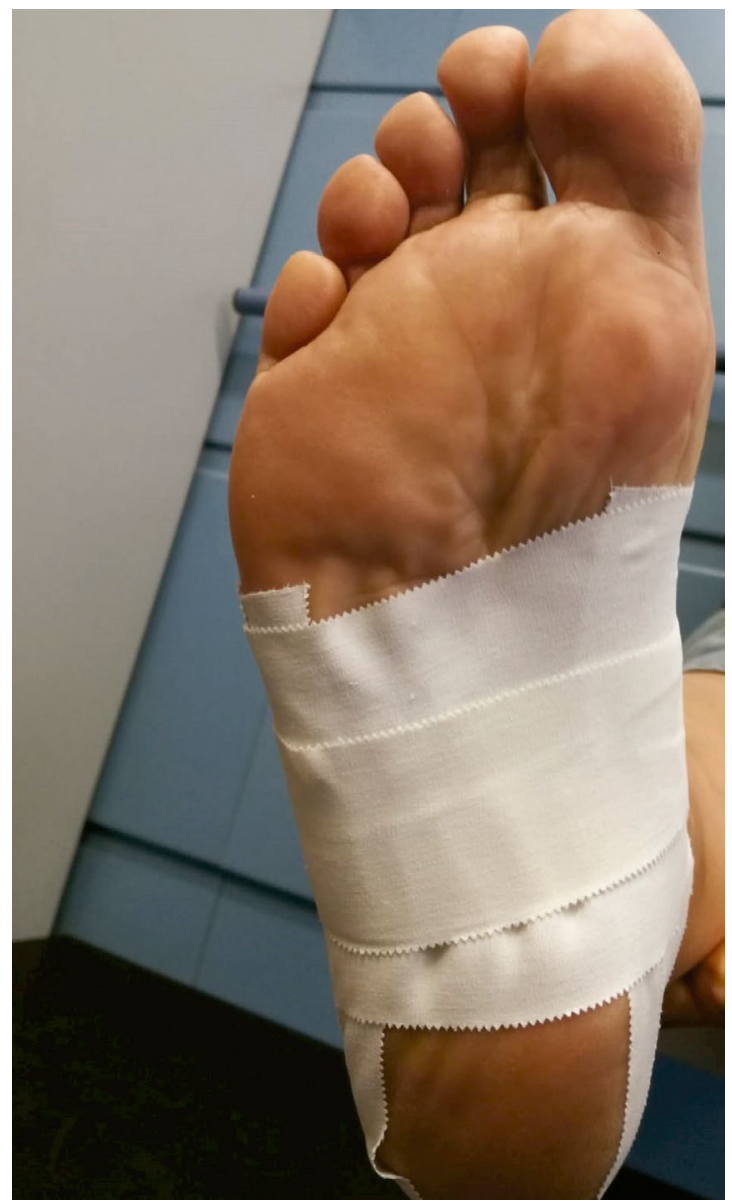

Figura 3. Vendaje Low-Dye. Tiras activas desde una visión plantar. 


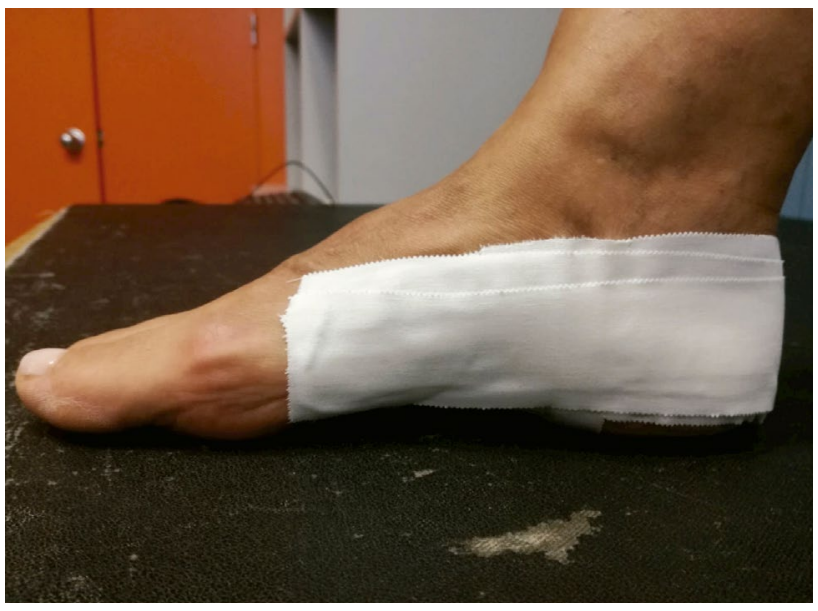

Figura 4. Vendaje Low-Dye. Tiras de cierre longitudinales desde una visión sagital.

según la tensión que apliquemos a la venda. Igualmente consiste en una técnica terapéutica ${ }^{6}$. El vendaje inelástico ha sido usado casi exclusivamente en el vendaje tipo Low-Dye con el fin de controlar la pronación del pie y disminuir el movimiento

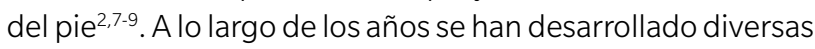
modificaciones de este vendaje como el vendaje tipo "LowDye aumentado" ${ }^{\prime 8,10}$. Actualmente es uno de los vendajes funcionales más utilizados en la práctica clínica por profesionales de la salud del pie. Numerosos estudios han abordados los efectos mecánicos generados por esta técnica de vendaje, sin embargo, ninguno de ellos ha relacionado los distintos resultados obtenidos entre si $^{1-10}$.

El objetivo principal de este trabajo consistió en realizar una revisión bibliográfica con el fin de evaluar los cambios mecánicos (cinéticos y cinemáticos) y clínicos (sobre determinadas patologías) que producen tanto el vendaje tipo Low-Dye como sus modificaciones (Low-Dye aumentado) sobre el pie y cómo estos cambios interactúan entre sí.

\section{METODOLOGÍA}

Para la elaboración del siguiente trabajo se llevó a cabo una revisión bibliográfica en las principales bases de datos científicas (PubMed, Web Of Science y Scopus) utilizando como términos de búsqueda: "Low-Dye tape", "Low-Dye taping" y "antipronation taping". La búsqueda fue realiza desde el 15 de septiembre de 2017 al 15 de diciembre de 2017.

Con dichos términos de búsqueda se obtuvieron un total de 699 resultados entre las tres bases de datos, incluyendo duplicados. Con el término "Low-Dye tape" se detuvieron 378 artículos, para el término "Low-Dye taping" se obtuvieron 304 resultados y, finalmente, para el término "antipronation taping" se obtuvieron 17 resultados.

Como criterios de inclusión para seleccionar los artículos que formarían parte de esta revisión se escogieron aque-

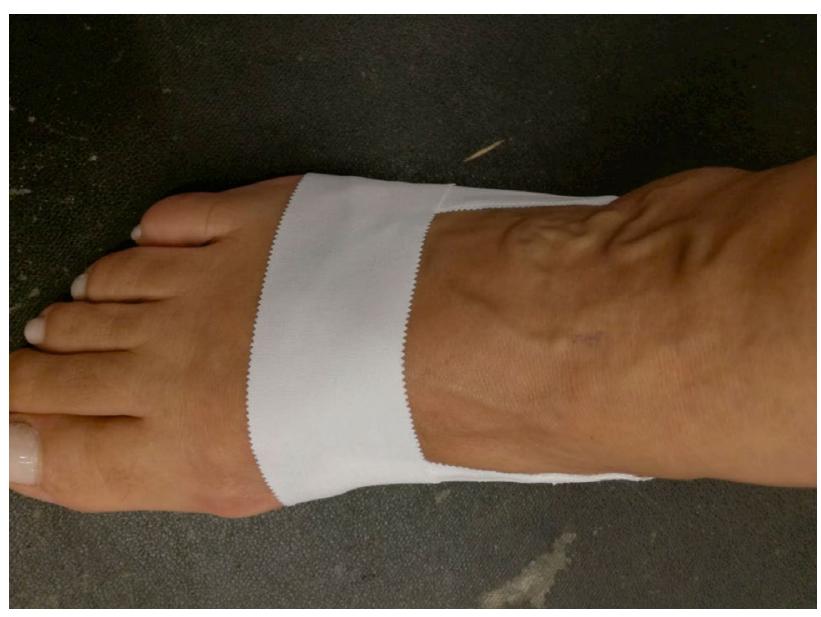

Figura 5. Vendaje Low-Dye. Tiras de cierre dorsales desde una visión dorsal.

Ilos ensayos clínicos, revisiones bibliográficas, revisiones sistemáticas y metanálisis publicados entre los años 2005 y 2017, que estuvieran escritos en inglés como idioma principal y que tuviesen como medida de resultado alguna de las variables mencionadas en el objetivo (cambios mecánicos y cambios en la evolución clínica de la patología en estudio).

Se excluyeron aquellos artículos donde no se mencionaba el término "Low-Dye tape". Esta selección se llevó a cabo mediante la lectura del título y del abstract.

Un solo revisor, y siempre el mismo (O. M. A.), llevó a cabo la selección de los artículos y el análisis crítico de los mismos.

\section{RESULTADOS}

De los 699 artículos encontrados, finalmente se seleccionaron 28 artículos (Tabla I) para la confección de nuestro trabajo tras aplicar los criterios de inclusión y exclusión y excluir los artículos duplicados en las tres bases de datos (Figura 6).

Efectos del vendaje tipo Low-Dye y sus modificaciones sobre la cinética del pie

A nivel cinético, los estudios realizados en los últimos 12 años se han centrado en la evaluación de las presiones plantares (PP) ${ }^{3,10-17}$. En nuestra revisión de la literatura recogimos un total de 9 artículos donde se estudiaron variables cinéticas. De esos 9 artículos, 7 fueron ensayos clínicos; 1 fue una revisión de la literatura y 1 fue una revisión sistemática (Tabla II).

Cronológicamente el primer artículo del cual obtenemos información es la revisión elaborada por Radford y cols. ${ }^{11}$ en el año 2006, donde no pudieron comparar los resultados de los tres ensayos clínicos recogidos acerca del estudio de las PP, debido a la gran heterogeneidad clínica (presencia de patología, tipo de pies, pacientes sedentarios o deportistas) 
Tabla I. Resumen de artículos incluidos en la revisión. Las variables de estudio se agrupan en función de los objetivos de esta revisión

\begin{tabular}{|c|c|c|c|c|}
\hline Título & Autor & Año & $\begin{array}{l}\text { Tipo de } \\
\text { estudio }\end{array}$ & Objetivo de estudio \\
\hline $\begin{array}{l}\text { Effectiveness of low-dye taping for the short-term } \\
\text { management of plantar fasciitis }\end{array}$ & Landorf y cols. & 2005 & Ensayo clínico & $\begin{array}{l}\text { Dolor en patología del } \\
\text { miembro inferior }\end{array}$ \\
\hline $\begin{array}{l}\text { Anti-pronation tape changes foot posture but not plantar } \\
\text { ground contact during gait }\end{array}$ & $\begin{array}{l}\text { Vicenzino y } \\
\text { cols. }\end{array}$ & 2006 & Ensayo clínico & Cinemática \\
\hline $\begin{array}{l}\text { The effect of Low-Dye Taping on Kinematic, Kinetic, and } \\
\text { Electromyographic Variables: A Systematic Review }\end{array}$ & Radford y cols. & 2006 & $\begin{array}{l}\text { Revisión } \\
\text { sistemática }\end{array}$ & $\begin{array}{l}\text { Cinética, cinemática y } \\
\text { neuromuscular }\end{array}$ \\
\hline $\begin{array}{l}\text { Effectiveness of low-Dye taping for the short-term treatment } \\
\text { of plantar heel pain: a randomised trial }\end{array}$ & Radford y cols. & 2006 & Ensayo clínico & $\begin{array}{l}\text { Dolor en patología del } \\
\text { miembro inferior }\end{array}$ \\
\hline $\begin{array}{l}\text { Treatment of plantar fascitis by LowDye taping and } \\
\text { iontophoresis: short term results of a double blinded, } \\
\text { randomised, placebo controlled clinical trial of } \\
\text { dexamethasone and acetic acid }\end{array}$ & Osborne y cols. & 2006 & Ensayo clínico & $\begin{array}{l}\text { Dolor en patología del } \\
\text { miembro inferior }\end{array}$ \\
\hline Cuboid syndrome: a review of the literature & Patterson & 2006 & $\begin{array}{l}\text { Revisión de la } \\
\text { literatura }\end{array}$ & $\begin{array}{l}\text { Dolor en patología del } \\
\text { miembro inferior }\end{array}$ \\
\hline $\begin{array}{l}\text { Plantar foot pressures after the augmented low dye taping } \\
\text { technique }\end{array}$ & $\begin{array}{l}\text { Vicenzino y } \\
\text { cols. }\end{array}$ & 2007 & Ensayo clínico & Cinética \\
\hline $\begin{array}{l}\text { The effect of low-dye taping on rearfoot and plantar pressure } \\
\text { during the stance phase of gait }\end{array}$ & $\begin{array}{l}\text { O' Sullivan y } \\
\text { cols. }\end{array}$ & 2008 & Ensayo clínico & $\begin{array}{l}\text { Cinética, cinemática y } \\
\text { neuromuscular }\end{array}$ \\
\hline $\begin{array}{l}\text { Tape that increases medial longitudinal arch height also } \\
\text { reduces leg muscle activity: a preliminary study }\end{array}$ & $\begin{array}{l}\text { Franettovich y } \\
\text { cols. }\end{array}$ & 2008 & Ensayo clínico & Neuromuscular \\
\hline $\begin{array}{l}\text { A physiological and psychological basis for anti-pronation } \\
\text { taping from a critical review of the literature }\end{array}$ & $\begin{array}{l}\text { Franettovich y } \\
\text { cols. }\end{array}$ & 2008 & $\begin{array}{l}\text { Revisión de la } \\
\text { literatura }\end{array}$ & $\begin{array}{l}\text { Cinética, cinemática y } \\
\text { neuromuscular }\end{array}$ \\
\hline $\begin{array}{l}\text { Use of antipronation taping to determine foot orthoses } \\
\text { prescription: a case series }\end{array}$ & Meier y cols. & 2008 & Ensayo clínico & $\begin{array}{l}\text { Prescripción ortesis } \\
\text { plantares }\end{array}$ \\
\hline $\begin{array}{l}\text { Effects of low-dye taping on plantar pressure pre and post } \\
\text { exercise: an exploratory study }\end{array}$ & Nolan y cols. & 2009 & Ensayo clínico & Cinética \\
\hline $\begin{array}{l}\text { Augmented Low Dye Taping Changes Muscle Activation } \\
\text { Patterns and Plantar Pressure During Treadmill Running }\end{array}$ & Kelly y cols. & 2010 & Ensayo clínico & $\begin{array}{l}\text { Cinética y } \\
\text { neuromuscular }\end{array}$ \\
\hline $\begin{array}{l}\text { Continual use of augmented low-dye taping increases arch } \\
\text { height in standing but does not influence neuromotor control } \\
\text { of gait }\end{array}$ & $\begin{array}{l}\text { Franettovich y } \\
\text { cols. }\end{array}$ & 2010 & Ensayo clínico & $\begin{array}{l}\text { Cinemática y } \\
\text { neuromuscular }\end{array}$ \\
\hline $\begin{array}{l}\text { Augmented Low-Dye tape alters foot mobility and } \\
\text { neuromotor control of gait in individuals with and without } \\
\text { exercise related leg pain }\end{array}$ & $\begin{array}{l}\text { Franettovich y } \\
\text { cols. }\end{array}$ & 2010 & Ensayo clínico & $\begin{array}{l}\text { Cinemática y } \\
\text { neuromuscular }\end{array}$ \\
\hline $\begin{array}{l}\text { Low-Dye taping versus medial arch support in managing pain } \\
\text { and pain-related disability in patients with plantar fascitis }\end{array}$ & $\begin{array}{l}\text { Abd El Salam y } \\
\text { cols. }\end{array}$ & 2011 & Ensayo clínico & $\begin{array}{l}\text { Dolor en patología del } \\
\text { miembro inferior }\end{array}$ \\
\hline $\begin{array}{l}\text { Immediate effects of a heel-pain orthosis and an augmented } \\
\text { low-dye taping on plantar pressures and pain in subjects with } \\
\text { plantar fasciitis }\end{array}$ & $\begin{array}{l}\text { Van Lunen y } \\
\text { cols. }\end{array}$ & 2011 & Ensayo clínico & $\begin{array}{l}\text { Cinética y dolor en } \\
\text { patología del miembro } \\
\text { inferior }\end{array}$ \\
\hline $\begin{array}{l}\text { A biomechanical analysis of the effects of low-Dye taping on } \\
\text { arch deformation during gait }\end{array}$ & Yoho y cols. & 2012 & Ensayo clínico & Cinética \\
\hline $\begin{array}{l}\text { Efficacies of different external controls for excessive foot } \\
\text { pronation: a meta-analysis }\end{array}$ & Cheung y cols. & 2012 & Metanálisis & Cinemática \\
\hline $\begin{array}{l}\text { A comparison of augmented low-Dye taping and ankle } \\
\text { bracing on lower limb muscle activity during walking in adults } \\
\text { with flat-arched foot posture }\end{array}$ & $\begin{array}{l}\text { Franettovich y } \\
\text { cols. }\end{array}$ & 2012 & Ensayo clínico & Neuromuscular \\
\hline
\end{tabular}




\begin{tabular}{|c|c|c|c|c|}
\hline Título & Autor & Año & $\begin{array}{l}\text { Tipo de } \\
\text { estudio }\end{array}$ & Objetivo de estudio \\
\hline Plantar fasciitis - an update & $\begin{array}{l}\text { Gautham y } \\
\text { cols. }\end{array}$ & 2014 & $\begin{array}{l}\text { Revisión de la } \\
\text { literatura }\end{array}$ & $\begin{array}{l}\text { Dolor en patología de } \\
\text { miembro inferior }\end{array}$ \\
\hline $\begin{array}{l}\text { Arch-Taping Techniques for Altering Navicular Height and } \\
\text { Plantar Pressures During Activity }\end{array}$ & Newell y cols. & 2015 & Ensayo clínico & Cinética y cinemática \\
\hline $\begin{array}{l}\text { The effects of the application of Low-Dye taping on paretic } \\
\text { side plantar pressure among patients with plantar fasciitis }\end{array}$ & Park y cols. & 2015 & Ensayo clínico & $\begin{array}{l}\text { Cinética y dolor en } \\
\text { patología de miembro } \\
\text { inferior }\end{array}$ \\
\hline $\begin{array}{l}\text { Effect of taping on foot kinematics in persons with chronic } \\
\text { ankle instability }\end{array}$ & $\begin{array}{l}\text { Deschamps y } \\
\text { cols. }\end{array}$ & 2015 & Ensayo clínico & Cinemática \\
\hline $\begin{array}{l}\text { Effects of the application of Low-Dye taping on the pain and } \\
\text { stability of patients with plantar fasciitis }\end{array}$ & Park y cols. & 2015 & Ensayo clínico & $\begin{array}{l}\text { Estabilidad postural y } \\
\text { equilibrio }\end{array}$ \\
\hline Taping for plantar fasciitis & Podolsky y cols. & 2015 & $\begin{array}{l}\text { Revisión de la } \\
\text { literatura }\end{array}$ & $\begin{array}{l}\text { Dolor en patología de } \\
\text { miembro inferior }\end{array}$ \\
\hline $\begin{array}{l}\text { Effects of Taping and Orthoses of Foot Biomechanics in Adults } \\
\text { with Flat-Arched Feet }\end{array}$ & Bishop y cols. & 2016 & Ensayo clínico & Cinemática \\
\hline $\begin{array}{l}\text { The Effectiveness of Physical Agents for Lower-Limb Soft } \\
\text { Tissue Injuries: A Systematic Review }\end{array}$ & Yu y cols. & 2016 & Ensayo clínico & $\begin{array}{l}\text { Dolor en patología de } \\
\text { miembro inferior }\end{array}$ \\
\hline
\end{tabular}

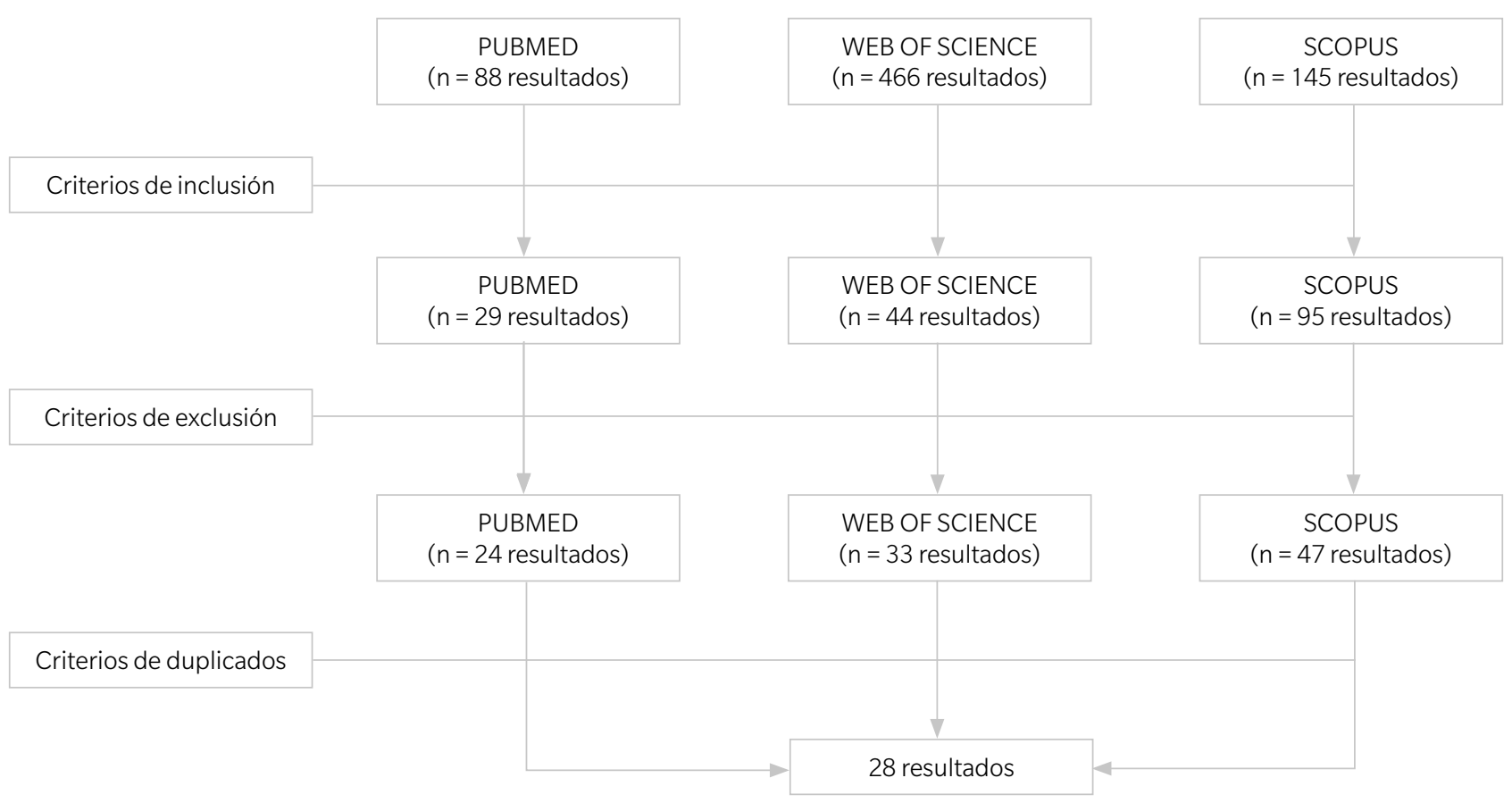

Figura 6. Diagrama de flujo de la búsqueda bibliográfica y aplicación de criterios de inclusión y exclusión.

y metodológica empleada por los estudios (diferentes plataformas de presiones plantares).

Los estudios realizados posteriormente a la revisión de Radford y cols. ${ }^{11}$ han contado en su mayoría con la participación de
20 o más sujetos, a excepción de tres trabajos ${ }^{12,13,18}$, que analizaron muestras inferiores a 20 individuos, siendo dichos autores los más recientes en nuestra revisión bibliográfica en evaluar la alteración de las PP mediante el vendaje tipo Low-Dye. 
Tabla II. Tabla resumen de artículos sobre efectos del vendaje Low-Dye en la cinética del pie

\begin{tabular}{|c|c|c|c|c|}
\hline Autor & Año & Tipo de estudio & $\begin{array}{c}\text { n. }{ }^{\circ} \text { pacientes / } \\
\text { artículos }\end{array}$ & Variables cinéticas de resultado \\
\hline Radford y cols. ${ }^{10}$ & 2006 & Revisión sistemática & 3 & $\begin{array}{l}\text { Área de superficie de contacto del pie } \\
\text { Media y pico de presiones plantares }\end{array}$ \\
\hline Vicenzino y cols. ${ }^{14}$ & 2007 & Ensayo clínico & 22 & Pico de presiones plantares y media de presión máxima \\
\hline O'Sullivan y cols. ${ }^{15}$ & 2008 & Ensayo clínico & 20 & Pico de presiones plantares \\
\hline Franettovich y cols. ${ }^{9}$ & 2008 & $\begin{array}{l}\text { Revisión de la } \\
\text { literatura }\end{array}$ & 6 & $\begin{array}{l}\text { Pico de presiones plantares } \\
\text { Fuerza de impacto del pie } \\
\text { Pico vertical de fuerzas de reacción del suelo }\end{array}$ \\
\hline Nolan y cols. ${ }^{11}$ & 2009 & Ensayo clínico & 12 & Pico de presiones plantares \\
\hline Kelly y cols. ${ }^{12}$ & 2010 & Ensayo clínico & 13 & Pico de presiones plantares \\
\hline Van Lunen y cols. ${ }^{13}$ & 2011 & Ensayo clínico & 17 & Pico y media de presiones plantares \\
\hline Newell y cols. ${ }^{3}$ & 2015 & Ensayo clínico & 25 & Pico de presiones plantares \\
\hline Park y cols. ${ }^{20}$ & 2015 & Ensayo clínico & 30 & Distribución anterior y posterior del peso \\
\hline
\end{tabular}

En 5 ensayos de los 7 incluidos en este apartado, se obtuvo un aumento de las PP en la región lateral del mediopié3,18-21 ,y en 4 de los 7 ensayos incluidos una disminución de las PP en la región medial del antepié ${ }^{3,19-21}$.

Otra posterior revisión de la literatura realizada por Franettovich y cols. incluyó 6 estudios (de los cuales 4 eran diferentes a los incluidos en la revisión realizada por Radford y cols.) que analizaron los efectos de un vendaje antipronador sobre las PP y sobre las fuerzas de impacto (2 emplearon la técnica de "Low-Dye aumentado" y 4 la técnica de vendaje Low-Dye) ${ }^{10}$.

Respecto a las presiones plantares, en dicha revisión se incluye el estudio de Russo y cols. ${ }^{21}$, quien observó que un vendaje Low-Dye disminuía el pico de PP en la región medial del antepié en un $20 \%$, mientras que aumentaban el pico de presiones en la región lateral del mediopié en un $26 \%$. Solo en el estudio de Sanzo y cols. ${ }^{22}$ se observó que el vendaje Low-Dye no producía cambios en las PP en la región del mediopié durante la marcha. Dos estudios fueron incluidos en la revisión ${ }^{10}$ que evaluaron las fuerzas de impacto del pie. En el estudio de Elizondo y cols. ${ }^{23}$ se investigó los efectos del vendaje Low-Dye en las fuerzas de impacto durante un salto en caída en individuos sanos, no observando cambios en el tiempo o en la magnitud del pico vertical de fuerzas de reacción del suelo con el vendaje. $Y$ en el estudio de Wall y cols. ${ }^{14}$ observaron un aumento en el tiempo del pico vertical de fuerzas de reacción del suelo durante un salto en caída en individuos con dolor en la espinilla con el vendaje.

Otro efecto cinético producto del vendaje Low-Dye consiste en un aumento de la distribución del peso en la región posterior del pie cuando el sujeto se encuentra en bipedestación. Esta observación es únicamente mencionada por Park y cols. ${ }^{15}$, quienes evaluaron los efectos a largo plazo del vendaje Low-Dye en las PP en 30 pacientes (2 grupos, uno con vendaje Low-Dye y otro control sin dicho vendaje) con fascitis plantar. Reportaron que la colocación del Low-Dye incrementó la presión que soportaban los sujetos en el retropié de forma significativa tanto al comparar con la situación previa sin vendaje como al comparar con el grupo control.

Por último, en 2 de los 7 ensayos clínicos incluidos se menciona la pérdida de los efectos del vendaje Low-Dye sobre las PP tras un periodo de tiempo de actividad ${ }^{3,12}$. En el caso de Newell y cols. ${ }^{3}$ se advirtió una pérdida de efectividad a los 15 minutos de la carrera, mientras que en el caso de Nolan y cols. ${ }^{12}$ se observó una pérdida de dichos efectos en un intervalo comprendido entre la primera sesión de 10 minutos y la segunda sesión de 20 minutos de la marcha; el único efecto conservado tras 20 minutos de marcha fue la tendencia del desplazamiento de las PP de medial a lateral.

Efectos del vendaje tipo Low-Dye y sus modificaciones sobre la cinemática del pie

En nuestra revisión recogimos un total de 11 artículos donde se estudiaron variables cinemáticas. De esos 11 artículos, 8 fueron ensayos clínicos, 1 fue una revisión de la literatura, 1 fue una revisión sistemática y 1 fue un metanálisis (Tabla III).

Para la investigación de los cambios cinemáticos, los estudios analizados valoraron 3 grupos de variables: varia- 
Tabla III. Tabla resumen de artículos sobre efectos del vendaje Low-Dye en la cinemática del pie

\begin{tabular}{|c|c|c|c|c|}
\hline Autor & Año & $\begin{array}{l}\text { Tipo de } \\
\text { estudio }\end{array}$ & $\begin{array}{l}\text { n. }{ }^{\circ} \text { pacientes } \\
/ \text { artículos }\end{array}$ & Variables a estudio \\
\hline Vicenzino y cols. ${ }^{27}$ & 2006 & Ensayo clínico & 18 & $\begin{array}{l}\text { - Ratio de la altura del arco del pie } \\
\text { - Área de contacto medial } \\
\text { - Índice del área lateral-medial }\end{array}$ \\
\hline Radford y cols. ${ }^{10}$ & 2006 & $\begin{array}{l}\text { Revisión } \\
\text { sistemática }\end{array}$ & 4 & $\begin{array}{l}\text { - Navicular Drop y altura del escafoides } \\
\text { - Movilidad de retropié }\end{array}$ \\
\hline O'Sullivan y cols. ${ }^{15}$ & 2008 & Ensayo clínico & 20 & Rango de movimiento articular \\
\hline Franettovich y cols. ${ }^{9}$ & 2008 & $\begin{array}{l}\text { Revisión de la } \\
\text { literatura }\end{array}$ & 11 & $\begin{array}{l}\text { - Eversión del calcáneo } \\
\text { - Altura del arco del pie }\end{array}$ \\
\hline Franettovich y cols. ${ }^{24}$ & 2010 & Ensayo clínico & 28 & $\begin{array}{l}\text { - Rango de movimiento articular } \\
\text { - Altura del arco plantar, ancho del mediopié, ratio de altura } \\
\text { del arco, diferencia de anchura del mediopié, la diferencia } \\
\text { de altura del arco plantar entre bipedestación y sedestación } \\
\text { y la magnitud de movilidad del pie }\end{array}$ \\
\hline Franettovich y cols. ${ }^{25}$ & 2010 & Ensayo clínico & 28 & $\begin{array}{l}\text { - Rango de movimiento articular } \\
\text { - Altura del arco plantar, ancho del mediopié y la magnitud de } \\
\text { movilidad del pie }\end{array}$ \\
\hline Yoho y cols. $^{2}$ & 2012 & Ensayo clínico & 21 & $\begin{array}{l}\text { - Arco longitudinal medial } \\
\text { - Índice de altura del arco }\end{array}$ \\
\hline Cheung y cols. ${ }^{21}$ & 2012 & Metanálisis & 6 & Eversión del calcáneo \\
\hline Newell y cols. ${ }^{3}$ & 2015 & Ensayo clínico & 25 & Altura del escafoides \\
\hline Deschamps y cols..$^{23}$ & 2015 & Ensayo clínico & 27 & Rango de movimiento articular \\
\hline Bishop y cols. ${ }^{26}$ & 2016 & Ensayo clínico & 21 & Rango de movimiento articular \\
\hline
\end{tabular}

bles relacionadas con la movilidad del retropié y el grado de eversión del calcáneo, variables relacionadas con el rango de movimiento articular (en primera articulación metatarsofalángica, articulación mediotarsiana, tobillo, rodilla, cadera y pelvis) y variables relacionadas con la altura del arco longitudinal interno del pie.

Entre los resultados, destacamos los obtenidos por O'SuIlivan y cols. ${ }^{22}$ en 2008, donde observaron una disminución en la movilidad del retropié tras la aplicación del vendaje, con una disminución del pico de pronación $(p=0.006)$ y de supinación ( $p=0.025)$. Posteriormente, tanto en la revisión realizada por Franettovich y cols. ${ }^{10}$, como en el metanálisis de Cheung y cols. ${ }^{16}$, este efecto es reforzado observándose una disminución en el pico de eversión del calcáneo.

En el metanálisis de Cheung y cols., donde contaron con 6 estudios (80 sujetos), observaron que la disminución en la eversión del calcáneo para las distintas intervenciones fue significativa en comparación con el grupo control no intervenido $(p<0.001)$. La disminución vista con el vendaje adhesivo terapéutico fue la mayor (diferencia media de $2.64^{\circ}$ ), seguido del calzado para control de movimiento (diferencia media de $2.52^{\circ}$ ) y por último las ortesis (diferencia media de $2.24^{\circ}$ ). Aun así, dentro de los vendajes, el vendaje tipo Low-Dye fue el que obtuvo una menor disminución en la eversión del calcáneo (diferencia media de $1.5^{\circ}$ ) en comparación con otras técnicas de vendaje como el High-Dye (Figuras 7-10), que fueron más efectivas en el control de la pronación (diferencia media de $\left.4.62^{\circ}\right)^{16}$. Contrario a los resultados anteriores, encontramos un estudio en la revisión de Radford y cols. ${ }^{11}$, donde no observaron cambios en la eversión máxima del retropié con el vendaje Low-Dye, aunque sí con el vendaje High-Dye ${ }^{17}$. Aun así, cabe decir que dicho hallazgo es anterior a los presentados por O'Sullivan y cols. ${ }^{22}$, Franettovich y cols. ${ }^{10}$ y Cheung y cols. ${ }^{16}$.

Deschamps y cols. investigaron el efecto del vendaje LowDye y High-Dye en la cinemática entre un grupo de personas sanas ( $n=12$ ) y sujetos con inestabilidad crónica de tobiIlo $(n=15)$ durante la carrera. En el grupo donde se aplicó el vendaje High-Dye observaron un aumento de la flexión dorsal del retropié (de un 38 a un $46 \%$ durante el ciclo de la carrera) en mayor medida que con el vendaje Low-Dye y también observaron una mayor inversión del ángulo formado entre el metatarsiano y el mediopié (de un $6 \%$ a un $24 \%$ durante el ciclo de la carrera) para el grupo donde se aplicó el vendaje Low-Dye en comparación con el grupo con inestabilidad crónica de tobillo donde no se aplicó ninguno 


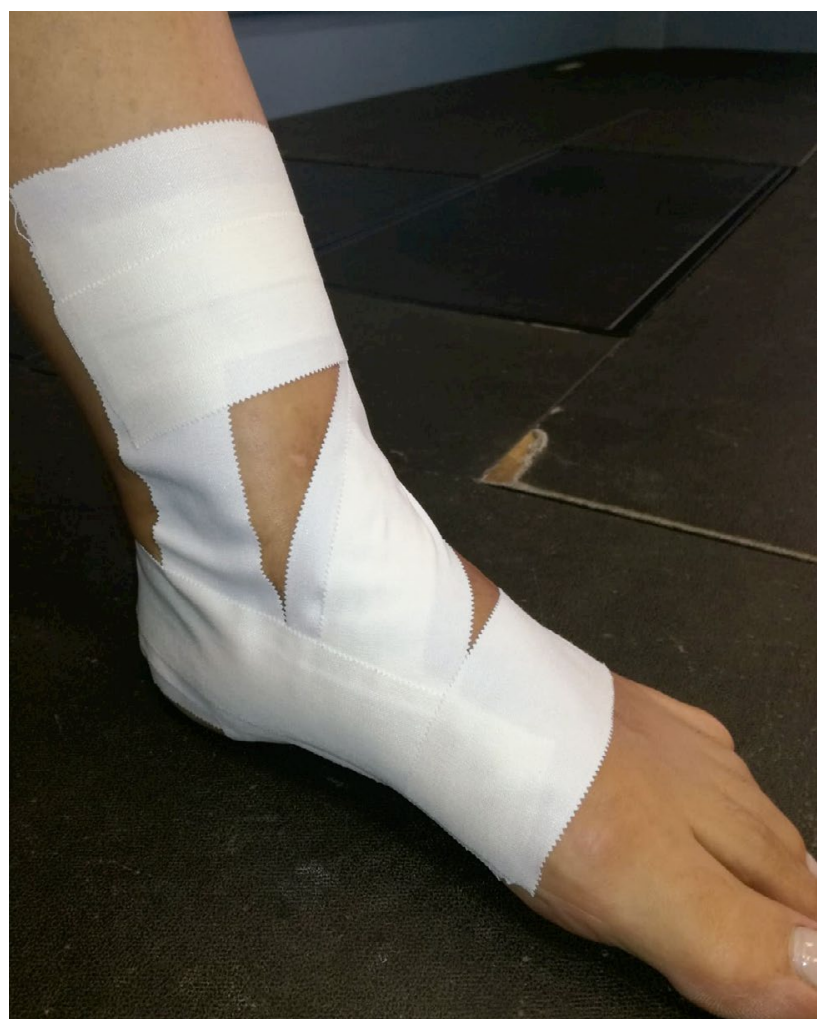

Figura 7. Vendaje High-Dye. Visión de vendaje High-Dye finalizado desde una visión lateral.

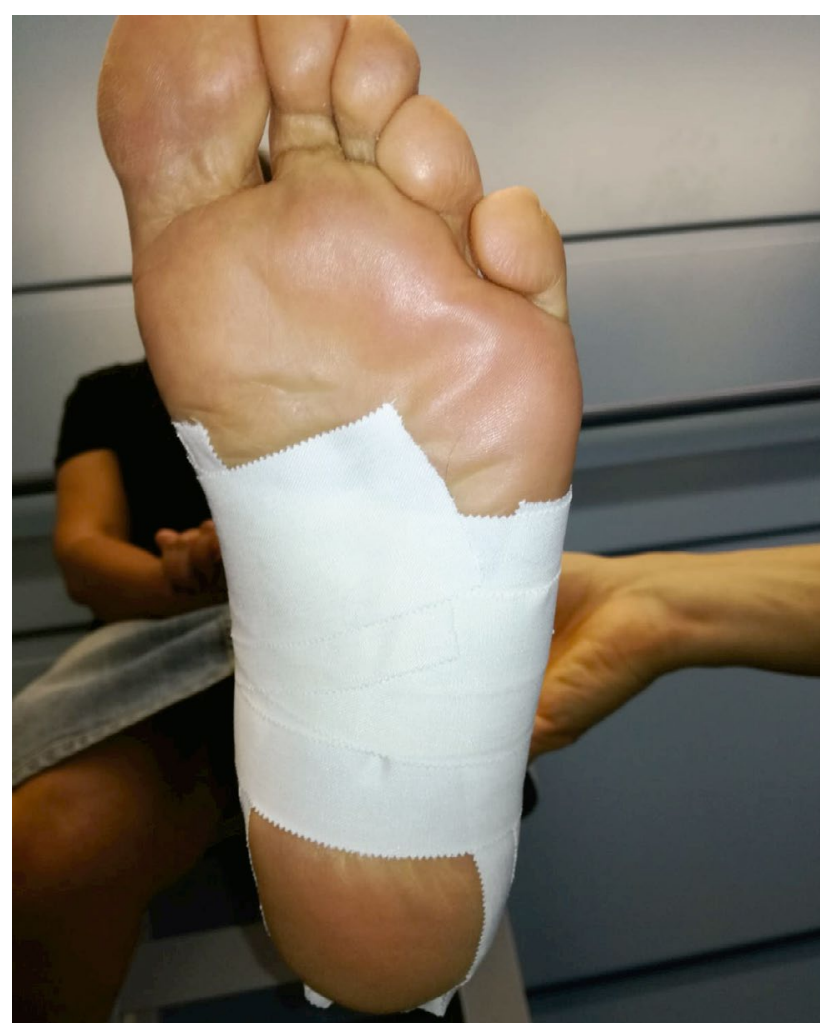

Figura 9. Vendaje High-Dye. Visión de vendaje High-Dye finalizado desde una visión plantar.

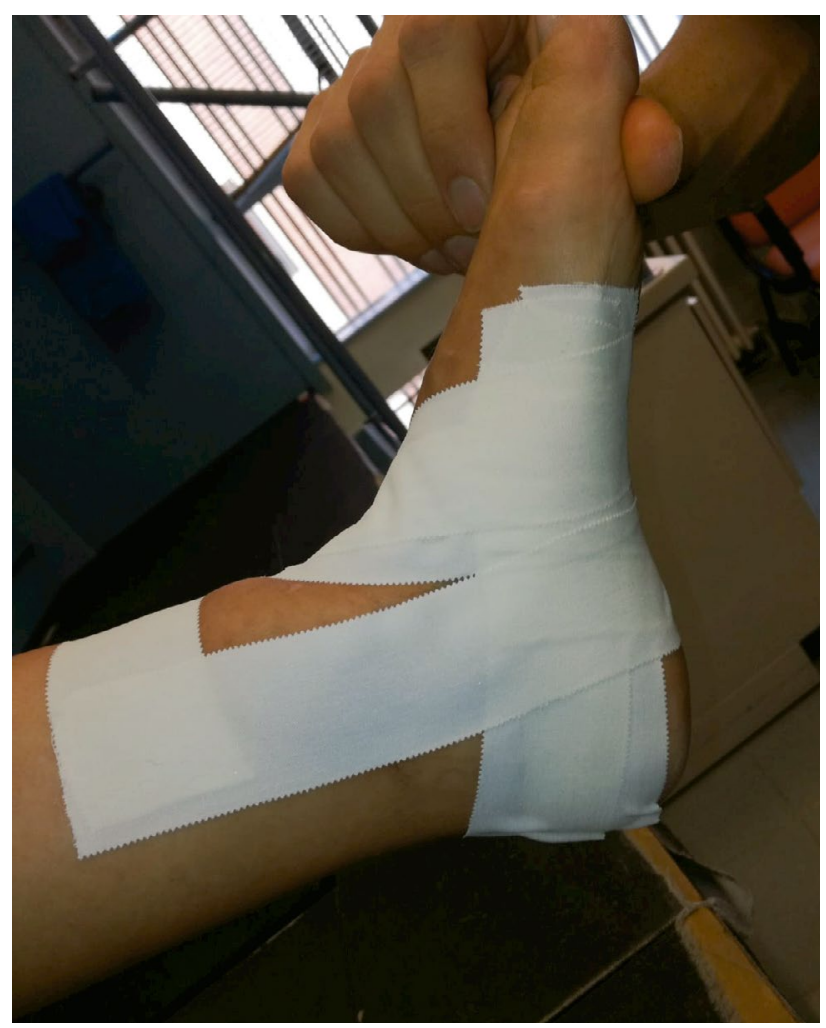

Figura 8. Vendaje High-Dye. Visión de vendaje High-Dye finalizado desde una visión sagital.

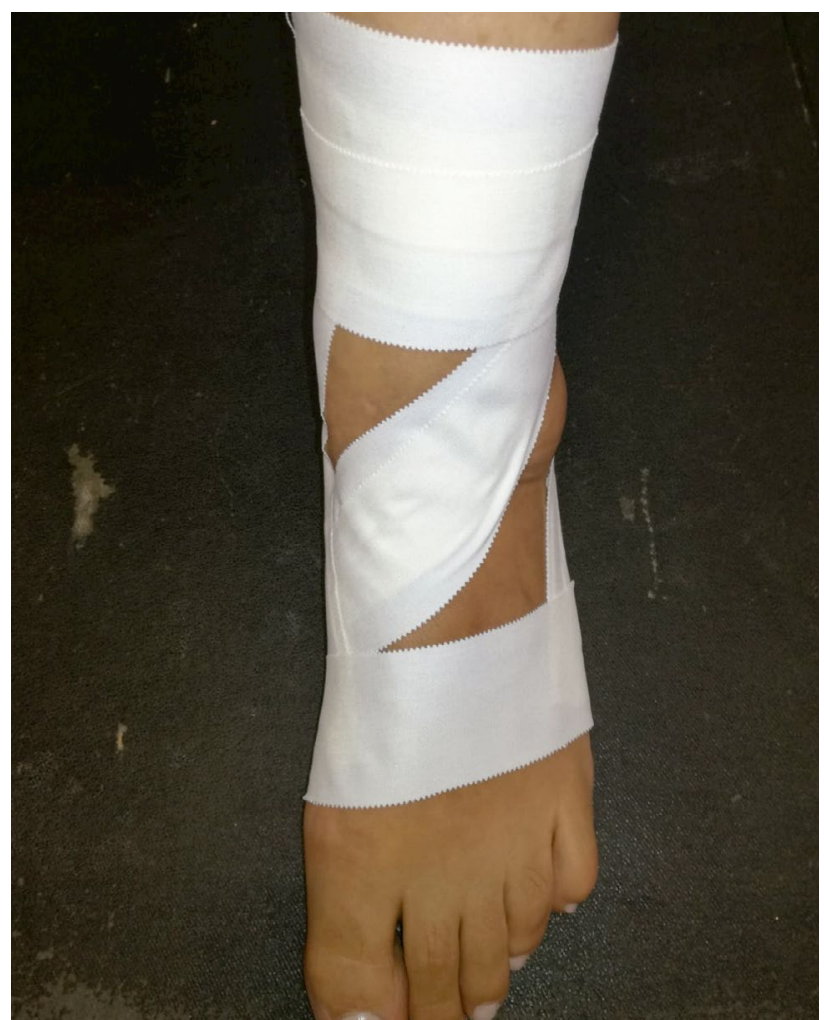

Figura 10. Vendaje High-Dye. Visión de vendaje High-Dye finalizado desde una visión dorsal. 
de los dos vendajes ${ }^{24}$.

En relación con el rango de movimiento articular, 5 artículos evaluaron los efectos del vendaje Low-Dye sobre diversas variables ${ }^{20,24-27}$. La comparación de dichos artículos entre sí es difícil, dado que cada estudio ha seguido una metodología propia, no obteniendo así resultados comparables entre sí.

En nuestra revisión, un total de 7 artículos evaluaron los efectos del vendaje Low-Dye sobre distintas variables relacionadas con la altura del arco longitudinal del pie, observando en todos ellos, una disminución en la caída del arco longitudinal del pie (referenciado también como un aumento en la altura del arco longitudinal del pie, un aumento en la altura del escafoides, una disminución en la caída del escafoides o una disminución en el navicular drop test) 2, 10,11,25,26,28. Tan solo en el caso de Newell y cols. observaron que con la aplicación inmediata del vendaje Low-Dye no había diferencias entre la altura del escafoides inicial y la inmediata tras la aplicación del vendaje $(p=0.30)$ o durante la carrera $(p>0.05)^{3}$.

Los estudios de Del Rossi y cols. ${ }^{29}$ (2004), y de Vicenzino y cols. $^{30}$ (1997) observaron que había un aumento de la altura del escafoides tras la aplicación del vendaje, efecto que se perdía después de la realización de ejercicio.

En 2010 Franettovich y cols., en un grupo de 28 participantes, observaron una ratio de la altura del arco plantar aumentado $(p=0.04)$ tras un periodo de 10 días de uso del vendaje "Low-Dye aumentado", no observándose dichos cambios para el grupo control $(p=0.36)$. La diferencia entre ambos grupos equivalía a un incremento de $0.16 \mathrm{~cm}$ en la altura del arco plantar para el grupo intervenido ${ }^{25}$. Ese mismo año, Franettovich y cols. llevaron a cabo otro estudio sobre un grupo de sujetos que debían caminar durante 10 minutos bajo 3 condiciones: antes del vendaje, vendado y después del vendaje; tras la retirada del vendaje, la altura de arco plantar del pie se mantuvo aumentado tanto en bipedestación $(0.09 \mathrm{~cm})$ como en sedestación $(0.11 \mathrm{~cm})$, así como la anchura del mediopié en bipedestación se mantuvo disminuida $(-0.10 \mathrm{~cm})^{26}$.

Posteriormente, Yoho y cols. (2012) evaluaron el arco longitudinal medial y el índice de altura del arco inmediatamente después de la colocación del vendaje y tras 48 horas de uso. Contaron con 21 sujetos sanos a los cuales se les aplicó el vendaje Low-Dye. Para el arco longitudinal medial hubo una disminución de un $19.3 \%$ inmediatamente después de la aplicación del vendaje $(p<0.02)$, aunque no se mantuvo tras 48 horas de uso del vendaje, habiendo solo una disminución de un $4.01 \%^{2}$.

Efectos del vendaje tipo Low-Dye y sus modificaciones sobre la estabilidad y control postural

El estudio de las presiones plantares mediante plataforma de presiones permite además evaluar variables relacionadas con la estabilidad y control postural.

En nuestra revisión de la literatura solo encontramos un artículo que respondiera a esta pregunta. Dicho artículo fue el ensayo clínico de Park y cols. ${ }^{5}$, quienes analizaron 30 sujetos, observando un aumento del área de transferencia del centro de gravedad en el grupo donde se aplicó el vendaje Low-Dye $(p<0.05)$. Dado que el único estudio que evaluó estas variables fue el de Park y cols. ${ }^{5}$, nos resultó imposible comparar sus resultados con los obtenidos por otros ensayos o revisiones, aunque hasta la realización de nuevos trabajos podría asumirse que el vendaje Low-Dye tiene un ligero efecto en la modificación del centro de gravedad.

\section{Efectos del vendaje tipo Low-Dye a nivel neuromuscular}

El estudio de la actividad neuromuscular también ha recibido atención por parte de la bibliografía. En nuestra revisión recogemos un total de 7 artículos donde se estudiaron variables electromiográficas relacionadas con la actividad muscular de diversos músculos del miembro inferior tras la aplicación del vendaje Low-Dye. De esos 7 artículos, 5 fueron ensayos clínicos, 1 fue una revisión de la literatura y 1 fue una revisión sistemática (Tabla IV).

En 3 de los 7 artículos incluidos se observó una disminución de la actividad muscular para los músculos tibial anterior y tibial posterior $r^{7,26,31}$.

Franettovich y cols. (2008) evaluaron los efectos iniciales del vendaje "Low-Dye aumentado" en la actividad muscular de los músculos tibial anterior, tibial posterior y peroneo lateral largo durante la marcha en 5 individuos asintomáticos con un arco plantar disminuido. Observaron una reducción del $23.9 \%$ y del $45.5 \%$ en el pico de activación del tibial anterior $y$ tibial posterior respectivamente $(p=0.003 ; p=0.02)$, pero no del peroneo largo $(p=0.561)^{7}$. En los estudios posteriores de Franettovich y cols. ${ }^{26,31}$ confirman dichos efectos, observándose, además, una disminución de la actividad muscular del gastrocnemio medial durante la fase de apoyo de la march ${ }^{26}$. En 2012 Franettovich y cols. compararon el efecto sobre la actividad muscular del vendaje "Low-Dye aumentado" frente a una tobillera semirrígida prefabricada comercial. Seleccionaron 27 individuos adultos sanos con un arco plantar aplanado. Observaron que el pico de activación para el tibial posterior disminuía un $22 \%$ con la tobillera $(p=0.004)$ y un $33,1 \%$ con el vendaje $(p<0.001)$ y para el tibial anterior en un $18.7 \%(p<0.001)$ y en un $13.1 \%(p=0.002)$ con la tobillera y el vendaje, respectivamente ${ }^{31}$.

En la revisión sistemática de Radford y cols. ${ }^{11}$ se incluye el trabajo de Boergers ${ }^{32}$, quien examinó la actividad del músculo tibial anterior durante la marcha y la carrera, viendo que el vendaje tipo Low-Dye disminuía la actividad muscular a la hora de correr; sin embargo, y en contraposición a los estudios de Franettovich y cols., la actividad muscular aumentaba durante la marcha. Estos resultados contradictorios podrían justificarse por el tipo de vendaje empleado. Franettovich y cols. emplearon la técnica "Low-Dye aumentado", la cual podría otorgar un mayor control cinético y cinemático de las estructuras articulares, llevando a cabo así una mayor reduc- 


\begin{tabular}{|c|c|c|c|c|}
\hline Autor & Año & $\begin{array}{l}\text { Tipo de } \\
\text { estudio }\end{array}$ & $\begin{array}{l}\text { n. }{ }^{\circ} \text { pacientes } \\
/ \text { artículos }\end{array}$ & Variables a estudio \\
\hline Radford y cols. ${ }^{10}$ & 2006 & $\begin{array}{l}\text { Revisión } \\
\text { sistemática }\end{array}$ & 1 & Tibial anterior \\
\hline Franettovich y cols. ${ }^{7}$ & 2008 & Ensayo clínico & 5 & Tibial anterior, tibial posterior y peroneo lateral largo \\
\hline Franettovich y cols. ${ }^{9}$ & 2008 & $\begin{array}{l}\text { Revisión de la } \\
\text { literatura }\end{array}$ & 3 & Tibial anterior, tibial posterior, peroneo lateral largo y sóleo \\
\hline Kelly y cols. ${ }^{12}$ & 2010 & Ensayo clínico & 13 & Vasto lateral, vasto medial y glúteo \\
\hline Franettovich y cols. ${ }^{24}$ & 2010 & Ensayo clínico & 28 & $\begin{array}{l}\text { Tibial anterior, tibial posterior, peroneo lateral largo, sóleo, } \\
\text { gastrocnemio medial y lateral, vasto lateral, vasto medial, recto } \\
\text { femoral, semitendinoso, bíceps femoral y glúteo medio }\end{array}$ \\
\hline Franettovich y cols. ${ }^{25}$ & 2010 & Ensayo clínico & 28 & $\begin{array}{l}\text { Tibial anterior, tibial posterior, peroneo lateral largo, sóleo, } \\
\text { gastrocnemio medial y lateral, vasto lateral, vasto medial, recto } \\
\text { femoral, semitendinoso, bíceps femoral y glúteo medio }\end{array}$ \\
\hline Franettovich y cols. ${ }^{30}$ & 2012 & Ensayo clínico & 27 & $\begin{array}{l}\text { Tibial anterior, tibial posterior, peroneo lateral largo y } \\
\text { gastrocnemio medial }\end{array}$ \\
\hline
\end{tabular}

ción en la actividad muscular.

Para el músculo peroneo lateral largo, 3 de los 7 artículos incluidos obtuvieron resultados significativos, aunque dichos resultados se confrontan entre sí, observando en el trabajo de Franettovich y cols. ${ }^{31}$ del año $2012(n=27)$ una disminución de la actividad muscular para el peroneo lateral largo en un $34 \%(p=0.019)$ y en un $29,4 \%(p=0.015)$ con el uso de una tobillera y el vendaje respectivamente, frente al estudio llevado a cabo por el mismo autor en el año $2010(n=28)$ donde observaron un aumento de la actividad de dicho músculo ${ }^{26}$. En el estudio de Wall y cols. ${ }^{14}$ observaron que el pico de actividad del músculo peroneo largo y sóleo disminuían con la aplicación de un vendaje "Low-Dye aumentado".

A nivel proximal, únicamente los trabajos de Kelly y cols. ${ }^{13}$ y Franettovich y cols. ${ }^{25,26}$, en 2010, han evaluado músculos localizados por encima de la articulación de la rodilla. Franettovich y cols. ${ }^{26}$ encontraron una disminución de la actividad muscular del vasto lateral. Sin embargo, estos cambios para el vasto lateral no mostraron diferencias con el grupo control en el estudio de Franettovich y cols. ( $p>0.05)$, lo cual podría interpretarse como variaciones naturales en los patrones de activación muscular ${ }^{25}$. Por su parte, Kelly y cols. ${ }^{13}$ sí encontraron que la colocación de un vendaje "Low-Dye aumentado" generaba un retraso en el tiempo de activación tanto de vasto lateral como de vasto medial y de glúteo medio $(p<0.05)$ cuando se comparaba con la ausencia de vendaje o la colocación de un vendaje de control de pronación que no incluía la articulación escafo-cuneana (las tiras de tape quedaban a la altura de tuberosidad escafoides).
Efectos del vendaje tipo Low-Dye en patologías del miembro inferior

En nuestra revisión de la literatura recogimos un total de 10 artículos donde se estudiaron variables relacionadas con el dolor y la función en el miembro inferior. De esos 10 artículos, 1 hablaba sobre el síndrome del cuboides, 8 de la fascitis plantar y 1 hablaba de patologías de partes blandas del miembro inferior, donde se incluía la fascitis plantar. De los 10 artículos, 6 fueron ensayos clínicos; 3 revisiones de la literatura y 1 fue una revisión sistemática (Tabla $\mathrm{V}$ ).

En todos los ensayos clínicos evaluados se observó una mejoría del dolor asociado a la fascitis plantar tras la colocación de un vendaje tipo Low-Dye.

Landorf y cols. (2005) evaluaron los efectos del vendaje Low-Dye en 105 participantes con fascitis plantar de, al menos, 4 semanas de evolución; 65 tratados mediante vendaje Low-Dye y 40 sin él. El dolor disminuyó en el grupo experimental $(44.0 \pm 17.9 \mathrm{~mm}$ inicial vs. $23.7 \pm 19.5 \mathrm{~mm}$ final $) \mathrm{a}$ las 2-3 semanas de tratamiento, mientras que para el grupo control aumentó ( $52.6 \pm 23.8 \mathrm{~mm}$ vs. $58.8 \pm 24.2)$ durante el periodo de intervención ${ }^{33}$. Ha y cols. ${ }^{34}$ (2012) mostraron una disminución inmediata del dolor tras aplicar tipo Low-Dye sobre 19 sujetos (media de 1,91 puntos, $\mathrm{p}<0.05$ ). Mientras que Park y cols. ${ }^{5}$ demostraron una disminución del dolor a largo plazo con el vendaje Low-Dye en comparación con el grupo control $(p<0.05)$.

Radford y cols., en otro ensayo clínico, compararon el vendaje Low-Dye frente a placebo en 92 participantes, pero aplicaron además en ambos grupos una terapia placebo simulando ultrasonidos. Observaron una mejoría en el dolor 
Tabla V. Tabla resumen de articulos sobre efectos del vendaje Low-Dye en el dolor en patologías del miembro inferior

\begin{tabular}{|c|c|c|c|c|}
\hline Autor & Año & Tipo de estudio & $\begin{array}{c}\text { n. }{ }^{\circ} \text { pacientes / } \\
\text { artículos }\end{array}$ & Variables a estudio \\
\hline Landorfy cols. ${ }^{33}$ & 2005 & Ensayo clínico & 105 & Dolor (fascitis) \\
\hline Radford y cols. ${ }^{35}$ & 2006 & Ensayo clínico & 92 & Dolor y función (fascitis) \\
\hline Osborne y cols. ${ }^{36}$ & 2006 & Ensayo clínico & 31 & Dolor y tensión fascial (fascitis) \\
\hline Abd El Salam y cols. ${ }^{6}$ & 2011 & Ensayo clínico & 30 & Dolor y discapacidad (fascitis) \\
\hline Van Lunen y cols. ${ }^{13}$ & 2011 & Ensayo clínico & 17 & Dolor (fascitis) \\
\hline Gautham y cols. ${ }^{37}$ & 2014 & Revisión de la literatura & No especifica & Dolor y función (fascitis plantar) \\
\hline Podolsky y cols. ${ }^{1}$ & 2015 & Revisión de la literatura & 5 & Dolor, función y discapacidad (fascitis) \\
\hline Park y cols. ${ }^{5}$ & 2015 & Ensayo clínico & 30 & Dolor (fascitis) \\
\hline Yu y cols. ${ }^{43}$ & 2016 & Revisión sistemática & 1 & Dolor y función (fascitis) \\
\hline Patterson ${ }^{38}$ & 2006 & Revisión de la literatura & No especifica & Dolor (síndrome del cuboides) \\
\hline
\end{tabular}

en los primeros pasos de la mañana para el grupo del vendaje en comparación con el grupo control con una diferencia de $-12.3 \mathrm{~mm}$ de media entre ambos grupos ( $p=0.017)$, aunque no se observaron diferencias en la función y el estado general de salud del pie, medido mediante el "Foot Health Status Questionnaire" ${ }^{35}$. Ese mismo año, Osborne y cols. ${ }^{36}$ mostraron mejoría del dolor matinal y de la tensión fascial matinal en un grupo de 31 pacientes tratados mediante la combinación de vendaje Low-Dye y iontoforesis de ácido acético y dexametasona.

En 2011 Abd El Salam y cols. ${ }^{7}$ también observaron una reducción del dolor $(p<0.05)$ y una mejora en el dolor relacionado con la discapacidad ( $p<0.05)$ medido mediante el cuestionario de "Manchester Foot Pain and Disability Schedule". Van Lunen y cols. observaron una disminución del dolor con el uso combinado del vendaje "Low-Dye aumentado" y la ortesis plantar para talalgia tanto en la marcha $(p=0.005$, $p=0.001$, respectivamente) como en la carrera $(p<0.001$, $p=0.002$, respectivamente) en comparación con la sesión donde no se aplicó ninguna de las dos intervenciones ${ }^{18}$.

Por último, respecto al síndrome del cuboides, Patterson ${ }^{37}$ menciona en su revisión de la literatura el uso del vendaje Low-Dye en aquellos casos donde el paciente quiere volver a realizar una actividad deportiva cuando sus dolores aún no se han resuelto, sumado o no a una almohadilla para el cuboides, aunque no se menciona si son capaces de reanudar su vida deportiva gracias al uso de dicho vendaje ${ }^{38-40}$. En nuestra revisión ningún otro trabajo menciona el uso de este vendaje para dicha patología, por lo que no podemos comparar los hallazgos mencionados por Patterson ${ }^{37}$ con otros trabajos y asegurar, por tanto, su efectividad en el manejo sintomatológico de esta entidad.

\section{Prescripción de ortesis plantares}

Dentro de nuestra revisión de la literatura, únicamente Meier y cols. (2008) evaluaron el uso de un vendaje tipo Low-Dye para determinar la prescripción de ortesis para el pie, quienes investigaron si un efecto positivo ante el uso de un vendaje en el pie podía ser indicativo de prescribir una ortesis funcional y si los cambios producidos por el vendaje podían usarse como guía en la prescripción de la ortesis plantar. Seleccionaron 7 atletas con dolor en la extremidad inferior de, al menos, 2 semanas de evolución, asociado a un exceso de pronación. Usaron dos vendajes, el vendaje tipo "Low-Dye aumentado" y el vendaje Reverse-6, para cada atleta. Tras tres días usando el vendaje se volvió a medir la altura del arco longitudinal del pie para la confección de las ortesis plantares, tomándose la diferencia en la altura del arco longitudinal del pie entre ambas mediciones como referencia para la adición de dicha diferencia de altura al arco longitudinal de unas ortesis prefabricadas. Después de llevar la ortesis plantar durante 4 semanas, el porcentaje de mejora en la escala del dolor osciló entre el 78 \% y el $100 \%$. La mejora en la escala para la función de FAAM fue de 2 a 11 puntos tras llevar el vendaje 3 días y de 5 a 19 puntos tras llevar la ortesis plantar durante 4 semanas ${ }^{41}$. Aunque está apoyado en los resultados 
de un único estudio, el empleo de un vendaje tipo Low-Dye podría permitirnos reproducir los gestos que posteriormente generará las ortesis plantar y evaluar de esta forma el pronóstico de esta. Pese a ello, sería necesario la realización de mayor número de trabajos para dar soporte a esta idea.

\section{DISCUSIÓN}

Efectos del vendaje tipo Low-Dye y sus modificaciones sobre la cinética del pie

A nivel cinético se observó una gran heterogeneidad metodológica de los estudios, debido al empleo de diversos métodos de medida (2 estudios emplearon el sistema F-Scan, 3 emplearon plataforma de presiones y 2 estudios emplearon el sistema in-shoe) y distinta metodología en la toma de las variables ( 2 estudios tuvieron en cuenta 6 áreas de análisis, 2 tuvieron en cuenta 7 áreas de análisis, 1 estudio tuvo en cuenta 5 áreas de análisis y 1 tuvo en cuenta 4 áreas de análisis), conduciendo así a una gran diversidad de resul$\operatorname{tados}^{3,10-13,15,18-20}$. De los 6 ensayos que evaluaron de forma específica las PP, y no otras variables cinéticas, 4 evaluaron las PP durante la marcha ${ }^{12,18-20}$ y 4 durante la carrera ${ }^{3,13,20,21}$. Este hecho ya es mencionado por Radford y cols. ${ }^{11}$ en su revisión sistemática del año 2006, algo que se mantiene en los trabajos posteriores a dicha revisión.

Para 5 de los 7 ensayos incluidos, las características de la población fueron homogéneas, seleccionando así sujetos sanos con un test de Navicular Drop positivo, 12,13,19,20. En los otros dos ensayos clínicos se seleccionaron sujetos que presentaban fascitis plantar ${ }^{15,18}$.

Tras exponer los resultados, parece existir una tendencia en los diferentes ensayos clínicos recogidos hacia una disminución de las PP en la región medial del antepié y su aumento en la región lateral del mediopié. Tan solo Nolan y cols. ${ }^{12}$ disciernen de estos hallazgos, mencionando en su estudio la obtención de un aumento de las PP a nivel medial en el antepié, aunque fue el estudio que menor muestra de sujetos recogió $(n=12)$. Existen indicios para pensar que con el paso del tiempo los cambios cinéticos van disminuyendo. Además, es probable que la selección de pacientes, mayoritariamente con un Navicular Drop positivo en la mayoría de los estudios, haga pensar que estos efectos cinéticos sean esperables en pacientes con una caída del escafoides superior a $8-10 \mathrm{~mm}$.

Efectos del vendaje tipo Low-Dye y sus modificaciones sobre la cinemática del pie

Bajo nuestro punto de vista estos hallazgos a nivel de PP guardarían relación con los resultados cinemáticos observados en la bibliografía donde, de nuevo, se observó una gran heterogeneidad en la metodología empleada por los diversos ensayos y estudios incluidos (especialmente en cuanto a las variables estudiadas, los métodos de medición de estas y el tipo de sujetos incluidos en los estudios), dificultando así la comparación de estos entre $\mathrm{si}^{2,3,10,11,16,20,24-28}$.

Cinco artículos contaron con sujetos sanos ${ }^{2,3,20,27,28}$ y 3 ensayos compararon un grupo sano con un grupo con patología ${ }^{24-26}$. De estos 8 ensayos, 3 tuvieron en cuenta un test de Navicular Drop positivo como criterio de inclusión ${ }^{3,20,28}$. En 2 de estos 3 estudios se consideró un Navicular Drop positivo como una diferencia en la caída del escafoides de más de $10 \mathrm{~mm}^{20,28}$ y en el otro que fuera más de $8 \mathrm{~mm}$. Siete ensayos emplearon sistema de cámaras ${ }^{2,20,24-28}$ para evaluar las variables cinemáticas a estudio y 1 empleó un calibre para medir el test de Navicular Drop ${ }^{3}$. Finalmente cabe mencionar que el único ensayo donde se evaluó como variable resultado de estudio el test de Navicular Drop corresponde al trabajo de Newell y cols. ${ }^{3}$.

A pesar de dicha heterogeneidad en las variables cinemáticas, podemos extraer algunas conclusiones, de modo que la literatura parece indicar que tras la colocación del vendaje Low-Dye se produce una disminución en la caída del arco longitudinal del pie y una disminución en el pico de eversión del calcáneo. Sin embargo, según la literatura, en cuanto a la disminución en el pico de eversión, el vendaje High-Dye es más eficaz que la técnica de vendaje Low-Dye. Existe contradicción sobre la persistencia de estos efectos en el tiempo.

Dentro de la cinemática, el estudio del arco longitudinal interno ha centrado la atención de un mayor número de autores, debido a la localización anatómica del vendaje Low-Dye. El principal efecto cinemático producto de la colocación del vendaje Low-Dye consiste en una disminución en la caída del arco longitudinal interno. Esta disminución en la caída del escafoides guardaría relación con los efectos cinemáticos observados recientemente por Bishop y cols. ${ }^{27}$ (2016), quienes observan una disminución de la flexión dorsal entre mediopié y antepié mediante el uso del vendaje Low-Dye dentro del calzado $(p<0.001)$. Este efecto podría interpretarse como que el vendaje Low-Dye genera un aumento de la rigidez a la flexión dorsal de la articulación escafo-cuneana y cuneo-metatarsiana. Además, dicho efecto cinemático explicaría otros gestos observados en la movilidad articular a nivel retrógrado tras la colocación de un vendaje Low-Dye, como son la disminución del pico de eversión y la disminución en la rotación tibial interna, como referencia Franettovich y cols. ${ }^{10}$. Bishop y cols. ${ }^{27}$ también mostraron que el vendaje Low-Dye aumentó el pico de flexión dorsal de la primera articulación metatarsofalángica $(p<0.001)$, el cual, según nuestro criterio, se explicaría como consecuencia de una disminución en el tiempo de instauración del mecanismo de Windlass como consecuencia del incremento de la rigidez a la flexión dorsal de las articulaciones escafo-cuneana y cuneo-metatarsiana.

Del mismo modo, los cambios cinemáticos producidos por el vendaje Low-Dye podrían explicar los cambios cinéticos mostrados anteriormente, de modo que el aumento de la rigidez a la flexión dorsal del primer radio explicaría una 
disminución de las presiones plantares en la región media del antepié y un aumento de estas en la región lateral del mediopié como consecuencia de una modificación en la posición del pie hacia la supinación.

\section{Efectos del vendaje tipo Low-Dye a nivel neuromuscular}

Respecto a los efectos neuromusculares, como en los apartados anteriores, volvemos a observar gran diversidad y heterogeneidad metodológica. Por ejemplo, respecto a las variables electromiográficas evaluadas, 4 de los 5 ensayos evaluaron el pico de actividad muscular, 3 de los 5 ensayos la duración y el comienzo de la actividad muscular, 2 de los 5 ensayos evaluaron la media, la media durante la fase de apoyo de la marcha, la media durante la fase de vuelo de la marcha, el fin y la amplitud de activación muscular. Pese a ello, sí hubo homogeneidad en la forma de medir la actividad muscular en los ensayos recogidos. En todos los estudios, para los músculos tibial anterior, sóleo, gastrocnemio medial, gastrocnemio lateral, vasto lateral, vasto medial, recto femoral, semitendinoso, bíceps femoral y glúteo medio, la técnica seleccionada consistió en electromiografía de superficie. De igual forma, para el músculo tibial posterior se empleó electromiografía intramuscular en todos los estudios. Para el peroneo lateral largo, sin embargo, encontramos que en el estudio de Franettovich y cols. ${ }^{8}$ de 2008 la técnica empleada fue electromiografía intramuscular, mientras que en sus trabajos posteriores ${ }^{25,26,31}$, la técnica fue de superficie.

A tenor de estos resultados, pensamos que el aumento de la rigidez a la flexión dorsal del primer radio explicaría la disminución de la actividad muscular de los músculos tibial anterior y tibial posterior, los cuales son los principales músculos extrínsecos encargados de controlar la caída del arco longitudinal del pie en cadena cinética cerrada mediante su contracción excéntrica. También podríamos explicar desde este punto de vista los resultados obtenidos sobre la disminución de la actividad muscular del músculo peroneo lateral largo, aunque este efecto ha mostrado resultados contradictorios en algunos estudios ${ }^{26,31}$. El incremento de la rigidez a la flexión dorsal (FD) del primer radio generaría una disminución de la magnitud de la contracción excéntrica del peroneo lateral largo en cadena cinética cerrada para frenar el desplazamiento en FD del primer radio.

Respecto a los resultados observados en grupos musculares más proximales a nivel de miembro inferior, los estudios han mostrado también un retardo en el tiempo de activación en glúteo medio y vasto medial que actúan como controladores del movimiento de rotación interna del miembro inferior y del momento valguizante que soporta la rodilla. Sin embargo, las limitadas referencia que hemos encontrado no dejan claro si dichos cambios pueden ser consecuencia de los cambios cinemáticos que genera el vendaje sobre el retropié (disminución del pico de eversión calcáneo) o pueden tener relación con una respuesta neuromuscular que regule el patrón de activación de los músculos durante la fase de oscilación de la marcha en función de las fuerzas que soporta el pie durante el paso anterior, como señala Nigg ${ }^{42}$.

Efectos del vendaje tipo Low-Dye en patologías del miembro inferior

Finalmente, a nivel de patología se observó de nuevo heterogeneidad en cuanto a los procedimientos realizados, observándose en 4 ensayos la combinación del vendaje Low-Dye con otras terapias como ultrasonidos, estiramientos o calzadoterapia ${ }^{6,33,35,36}$. Pese a ello, se observa gran homogeneidad en los resultados obtenidos pudiendo concluir que, tanto en los distintos ensayos realizados, como en las revisiones incluidas, se observó una mejoría del dolor, la función y la discapacidad relacionada con el dolor de fascitis plantar mediante el uso del vendaje Low-Dye 1,5,7,18,33,35,36,43.

Se ha teorizado que la disminución del dolor se basa en la reducción de la pronación del pie, produciendo una elevación y un soporte del arco longitudinal interno que reduciría la tensión a lo largo de la fascia plantar ${ }^{7}$. Estamos de acuerdo en esta hipótesis, puesto que los efectos cinéticos y cinemáticos mencionados anteriormente, favorecen la presencia de un arco interno de mayor altura y una mayor rigidez al movimiento de flexión dorsal de las articulaciones que componen la columna medial. En esta situación se produce una menor demanda de tensión de la fascia plantar, dado que se incrementa su brazo de momento en relación con las articulaciones de la columna interna (distancia entre fascia y el eje de rotación de las articulaciones).

Cuando se mencionaron anteriormente los efectos neuromusculares advertíamos una disminución de la actividad muscular del tibial anterior y del tibial posterior. Por tanto, es lógico pensar que esto conduciría a una mejora de la sintomatología producida por patologías asociadas con dichos músculos y tendones. Sin embargo, en nuestra revisión bibliográfica no recogimos ningún artículo que haya evaluado el grado de mejoría en el dolor y la función estos músculos con el uso de un vendaje tipo Low-Dye o sus modificaciones. Así, consideramos este hallazgo de interés a la hora de realizar futuros estudios. Incluso este hecho podría ser extrapolable a otras patologías localizadas a niveles más craneales, como el síndrome de estrés tibial medial o síndromes patelofemorales.

\section{CONCLUSIÓN}

Cinéticamente, el vendaje Low-Dye produce una disminución de las presiones plantares en la región medial del antepié y un aumento de estas en la región lateral del mediopié. Este efecto cinético se relacionaría con los efectos cinemáticos producto de un vendaje tipo Low-Dye, los cuales se caracterizan por una disminución de la caída del escafoides, una disminución en el pico de eversión del calcáneo, una disminución en la rotación tibial interna, una disminución de la flexión 
dorsal de antepié sobre mediopié y un aumento del rango de flexión dorsal de la primera articulación metatarsofalángica. Todos los cambios cinemáticos indican que el vendaje Low-Dye disminuye el momento de fuerza pronador neto que soporta el pie, así como el momento de flexión dorsal neto que soportan las articulaciones de la columna interna y podría favorecer la instauración del mecanismo de Windlass. Como consecuencia de estos cambios cinéticos y cinemáticos, se reduce, principalmente, la actividad muscular para los músculos tibial anterior y tibial posterior, estructuras encargadas de controlar el exceso de pronación del pie y la caída del escafoides; y se reduce la tensión fascial de la aponeurosis plantar. Por sus efectos cinéticos y cinemáticos, esta técnica de vendaje se muestra, en base a nuestra revisión narrativa, como una herramienta útil en el manejo temporal de la fascitis plantar. Tomando como referencia los cambios cinemáticos observados con la colocación del vendaje Low-Dye, otras patologías que tienen su base patomecánica en el incremento de momentos de fuerza pronadores (por ejemplo la tendinopatía del tendón tibial posterior) podrían beneficiarse de su aplicación. Sin embargo, no se recogen en la literatura trabajos que evalúen si este vendaje contribuye a disminuir la clínica dolorosa de estos pacientes.

\section{CONFLICTO DE INTERESES}

Los autores declaran que no presentan ningún conflicto de intereses relevante en este artículo.

\section{FINANCIACIÓN}

No existen fuentes de financiación públicas o privadas en la realización del presente estudio.

\section{BIBLIOGRAFÍA}

1. Podolsky R, Kalichman L. Taping for plantar fasciitis. J Back Musculoskelet Rehabil. 2015;28(1):1-6. DOI: 10.3233/BMR-140485.

2. Yoho R, Rivera JJ, Renschler R, Vardaxis VG, Dikis J. A biomechanical analysis of the effects of low-Dye taping on arch deformation during gait. Foot. 2012;22(4):283-6. DOI: 10.1016/j.foot.2012.08.006.

3. Newell T, Simon J, Docherty CL. Arch-Taping Techniques for Altering Navicular Height and Plantar Pressures During Activity. J Athl Train. 2015;50(8):825-32. DOI: 10.4085/1062-6050-50.5.05

4. Dye RW. A strapping. J Natl Assoc Chiropodists. 1939;29:11-12.

5. Park C, Lee S, Lim DY, Yi CW, Kim JH, Jeon C. Effects of the application of Low-Dye taping on the pain and stability of patients with plantar fasciitis. J Phys Ther Sci. 2015;27(8):2491-3. DOI: 10.1589/jpts.27.2491.

6. Bové T. El vendaje functional. $6^{\mathrm{a}}$ edición. Madrid: Elsevier; 2015.

7. Abd El Salam MS, Abd Elhafz YN. Low-Dye taping versus medial arch support in managing pain and pain-related disability in patients with plantar fascitis. Foot Ankle Spec. 2011;4(2):86-91. DOI: 10.1177/1938640010387416

8. Franettovich MM, Chapman A, Vicenzino B. Tape that increases medial longitudinal arch height also reduces leg muscle activity: a preliminary study. Med Sci Sports Exerc. 2008;40(4):593-600. DOI: 10.1249/ MSS.0b013e318162134f.

9. Hadley A, Griffiths SR, Griffiths LA, Vicenzino B. Antipronation taping and temporary orthoses. Effects on tibial rotation position after exercise. J Am Pod Med Assoc. 1999;89(3):118-23.
10. Franettovich MM, Chapman A, Blanch P, Vicenzino B. A physiological and psychological basis for anti-pronation taping from a critical review of the literature. Sports medicine (Auckland, NZ). 2008;38(8):617-31.

11. Radford JA, Burns J, Buchbinder R, Cook C. The effect of Low-Dye Taping on Kinematic, Kinetic, and Electromyographic Variables: A Systematic Review. J Orthop Sports Phys Ther. 2006;36(4):232-41. DOI: 10.2519/ jospt.2006.36.4.232.

12. Nolan $D$, Kennedy N. Effects of low-dye taping on plantar pressure pre and post exercise: an exploratory study. BMC Musculoskelet Disord. 2009;10:40. DOI: 10.1186/1471-2474-10-40.

13. Kelly LA, Racinais S, Tanner CM, Grantham J, Chalabi H. Augmented Low Dye Taping Changes Muscle Activation Patterns and Plantar Pressure During Treadmill Running. J Orthop Sports Phys Ther. 2010;40(10):64855. DOI: 10.2519/jospt.2010.3164.

14. Wall K, Swanik K, Swanik C, et al. Augmented low-dye arch taping effects on muscle activity and ground reaction forces in people with pes planus. J Athlet Train. 2005;40(2):S-97.

15. Park C, Lee S, Lim DY, Kim S, Hwangbo G. The effects of the application of Low-Dye taping on paretic side plantar pressure among patients with plantar fasciitis. J Phys Ther Sci. 2015;27(11):3555-7. DOI: 10.1589/ jpts.27.3555.

16. Cheung RT, Chung RC, Ng GY. Efficacies of different external controls for excessive foot pronation: a meta-analysis. Br J Sports Med. 2011;45(9):743-51. DOI: 10.1136/bjsm.2010.079780.

17. Keenan AM, Tanner CM. The effect of high-Dye and low-Dye taping on rearfoot motion. J Am Podiatr Med Assoc. 2001;91:255-61.

18. Van Lunen B, Cortes N, Andrus T, Walker M, Pasquale M, Onate J. Immediate effects of a heel-pain orthosis and an augmented low-dye taping on plantar pressures and pain in subjects with plantar fasciitis. Clin J Sport Med. 2011;21(6):474-9. DOI: 10.1097/JSM.0b013e3182340199.

19. Vicenzino B, McPoil T, Buckland S. Plantar foot pressures after the augmented low dye taping technique. J Athl Train. 2007;42(3):374-80.

20. O'Sullivan K, Kennedy N, O'Neill E, Ni Mhainin U. The effect of low-dye taping on rearfoot and plantar pressure during the stance phase of gait. BMC Musculoskelet Disord. 2008;9:111. DOI: 10.1186/1471-2474-9-111.

21. Russo SJ, Chipchase LS. The effect of low-Dye taping on peak plantar pressures of normal feet during gait. Aust J Physiother. 2001;47(4):239.

22. Sanzo P, Bauer T. The effects of low dye taping on foot pressure in subjects with plantar fasciitis. Proceedings of the XIII International Symposium for Biomechanics in Sport; 1996 Jul 18-22; Thunder Bay (ON): Lakehead University, 1996:13-5.

23. Elizondo M, Wojciechowski R, Cordova M, et al. The effects of arch taping on vertical ground reaction forces generated during a drop landing. J Athelet Train. 2004;39 Suppl. 2:101.

24. Deschamps K, Dingenen B, Pans F, Van Bavel I, Matricali GA, Staes F. Effect of taping on foot kinematics in persons with chronic ankle instability. J Sci Med Sport. 2016;19(7):541-6. DOI: 10.1016/j. jsams.2015.07.015.

25. Franettovich M, Chapman A, Blanch P, Vicenzino B. Continual use of augmented low-dye taping increases arch height in standing but does not influence neuromotor control of gait. Gait Posture. 2010;(2):24750. DOI: 10.1016/j.gaitpost.2009.10.015.

26. Franettovich M, Chapman AR, Blanch P, Vicenzino B. Augmented LowDye tape alters foot mobility and neuromotor control of gait in individuals with and without exercise related leg pain. J Foot Ankle Res. 2010;3:5. DOI: 10.1186/1757-1146-3-5.

27. Bishop C, Arnold JB, May T. Effects of Taping and Orthoses of Foot Biomechanics in Adults with Flat-Arched Feet. Med Sci Sports Exerc. 2016;48(4):689-96. DOI: 10.1249/MSS.0000000000000807.

28. Vicenzino B, McPoil TG, Russell T, Peisker S. Anti-pronation tape changes foot posture but not plantar ground contact during gait. Foot. 2006;16(2):91-7.

29. Del RG, Fiolkowski P, Horodyski MB, Bishop M, Trimble M. For how long do temporary techniques maintain the height of the medial longitudinal arch? Phys Ther Sport. 2004;5:84-9.

30. Vicenzino B, Feilding J, Howard R, Moore S, Smith S. An investigation of the anti-pronation effect of two taping methods after application and exercise. Gait Posture. 1997;5:1-5. 
31. Franettovich MM, Murley GS, David BS, Bird AR. A comparison of augmented low-Dye taping and ankle bracing on lower limb muscle activity during walking in adults with flat-arched foot posture. J Sci Med Sport. 2012;15(1):8-13. DOI: 10.1016/j.jsams.2011.05.009.

32. Boergers RJ. Effects of Arch Taping on Peak Force, Surface Contact Area and Neuromuscular Activity at Midstance [dissertation]. Wisconsin, IL: University of Wisconsin-La Crosse; 2000.

33. Landorf KB, Radford JA, Keenan AM, Redmond AC. Effectiveness of low-dye taping for the short-term management of plantar fasciitis. J Am Podiatr Med Assoc. 2005;95:525-30.

34. Ha HS, Hwang JH, Kim YB, Ha YM, Hwang JM. Immediate Clinical and biomechanical effects of low-dye taping in patients with plantar heel pain. Korean J Sports Med. 2012;30:9-15.

35. Radford JA, Landorf KB, Buchbinder R, Cook C. Effectiveness of low-Dye taping for the short-term treatment of plantar heel pain: a randomised trial. BMC Musculoskelet Disord. 2006;7:64.

36. Osborne HR, Allison GT. Treatment of plantar fascitis by LowDye taping and iontophoresis: short term results of a double blinded, randomised, placebo controlled clinical trial of dexamethasone and acetic acid. $\mathrm{Br}$ J Sports Med. 2006;40(6):545-9. DOI: 10.1136/bjsm.200.5.021758.

37. Patterson SM. Cuboid syndrome: a review of the literature. J Sports Sci Med. 2006;5(4):597-606.

38. Newell SG, Woodle A. Cuboid Syndrome. Phys Sports Med. 1981;9:71-6.

39. Blakeslee TJ, Morris JL. Cuboid syndrome and the significance of midtarsal joint stability. J Am Podiatr Med Assoc. 1987;77(12):638-42. DOI: 10.7547/87507315-77-12-638

40. Marshall P, Hamilton WG. Cuboid subluxation in ballet dancers. Am J Sports Med. 1992;20(2):169-75. DOI: 10.1177/036354659202000213.

41. Meier K, McPoil TG, Cornwall MW, Lyle T. Use of antipronation taping to determine foot orthoses prescription: a case series. Res Sports Med. 2008;16(4):257-71. DOI: 10.1080/15438620802310842.

42. Nigg BM. The role of impact forces and foot pronation: a new paradigm. Cli J Sport Med. 2001;11(1):2-9

43. Gautham P, Nuhmani S, Kachanathu SJ. Plantar fasciitis - an update. Bangladesh Journal of Medical Science. 2015;14(01): 3-8. 